\title{
Avaliação da pectina fosfatada aplicada na formação de filmes isolados. Material candidato a novos sistemas para liberação modificada de fármacos
}

\author{
João Philype Andrade Souto-Maior', Adriano Valim Reis' ${ }^{2}$, Liliane Neves Pedreiro', \\ Osvaldo Albuquerque Cavalcanti ${ }^{1 *}$ \\ ${ }^{1}$ Laboratório de Tecnologia Farmaceutica, Departamento de Farmácia e Farmacologia, \\ Universidade Estadual de Maringá \\ ${ }^{2}$ Departamento de Química, Universidade Estadual de Maringá
}

*Correspondência:

O. A. Cavalcanti

Departamento de Farmácia e

Farmacologia

Laboratório de Tecnologia

Farmacêutica

Universidade Federal de Maringá Avenida Colombo 5790, Anexo Bloco P0 2

87020-900 - Maringá-PR, Brasil

E-mail: oacavalcanti@uem.br
Pectina fosfatada (Pect-TMFT) juntamente com o $\alpha$-Glucooligossacarídeo (Bioecolians ${ }^{\circledR}$ ) foram incorporados à dispersão aquosa de polimetacrilato (Eudragit ${ }^{\circledR} R S 30$ D) para obtenção de filmes isolados pelo processo "casting" $\left(50^{\circ} \mathrm{C}\right)$ em placa de Teflon ${ }^{\circledR}$. A Pect-TMFT e o Bioecolians ${ }^{\circledR}$ foram adicionados à dispersão de Eudragit ${ }^{\circledR}$ RS 30 D nas concentrações: 90: 05: 05, 80: 10: 10 e 70: 20: $10(4 \% \mathrm{p} / \mathrm{v})$. Citrato de trietila (TEC) foi utilizado como plastificante ( $20 \%$ da massa do polimetacrilato). As dispersões propostas apresentaram habilidades filmogênicas. Os filmes isolados foram caracterizados pela determinação da permeabilidade ao vapor d'água (TVA), pelo índice de intumescimento ( $\left.I_{i} \%\right)$ em fluidos de simulação gástrica (FSG) e intestinal (FSI) e por microscopia eletrônica de varredura (MEV). $O$ aumento do polissacarídeo modificado e do Bioecolians ${ }^{\circledR}$ nos filmes favoreceram a permeabilidade ao vapor d'água e o grau de hidratação quando em FSI $(p H=6,8)$. Dessa forma, o filme 70: 20: 10, poderá impedir a liberação prematura do fármaco em regiões superiores do TGI quando aplicado como revestimento de sistemas sólidos orais. Além disso, a presença da Pect-TMFT e do Bioecolians ${ }^{\circledR}$, poderá favorecer a degradação específica da película por enzimas produzidas pela microflora colonica, possibilitando uma cinética de liberação modificada de fármacos apesar das variações inter-individuais de pH que possam existir.
Unitermos

- Pectina fosfatada

- Filmes isolados

- $\alpha$-Gluco-oligossacarídeo

- Eudragit ${ }^{\circledR}$ RS 30 D

- Fármacos/Liberação modificada

\section{INTRODUÇÃO}

Inúmeras patologias requerem tratamentos com longos esquemas posológicos e elevadas doses de fármacos. Estes tratamentos podem gerar uma série de efeitos colaterais e/ou reações adversas, comportamento inerente prioritariamente, às formas farmacêuticas convencionais. Neste contexto merece destaque a forma sólida para administração oral, 
uma vez que não é invasiva, apresenta menor custo, simplicidade no processo de fabricação e também no que se refere aos equipamentos e instalações. Entretanto, a administração oral de fármacos nem sempre é viável, especialmente para aqueles oriundos da biotecnologia, pois estes produtos apresentam elevada susceptibilidade diante das exigências fisiológicas do trato gastrintestinal (Cavalcanti et al., 2002; Ahmed et al., 2002; Liu et al., 2003; Ibekwe et al., 2006).

Desencadear pesquisas voltadas à promissora perspectiva em veicular fármacos às regiões específicas do trato gastrointestinal (TGI) constitui desafio capaz de permitir efetivo controle da liberação, harmonizando efeitos terapêuticos. Produtos destinados ao tratamento de patologias que afetam a saúde humana e animal encontram, neste contexto, elevada expectativa para administração via oral, especialmente para tratamentos crônicos. Genes, oligonucleotídeos e vacinas, baseados em proteínas e polipeptídios representam categoria de substâncias disponíveis atualmente pelas indústrias farmacêuticas e de biotecnologia que encontram certas restrições de uso diante da sua elevada susceptibilidade a enzimas digestivas gástricas (proteolíticas), baixa absorção aliada à capacidade limitada para atravessar a barreira do epitélio intestinal (Ahmed et al., 2002; Liu et al., 2003; Irache et al., 2005, Dupuis et al., 2006).

$\mathrm{Na}$ tentativa de resguardar a integridade e garantir a eficácia terapêutica dessas promissoras moléculas observamos crescente interesse na $\mathrm{P} \& \mathrm{D}$ voltada ao delineamento de novas estratégias, as quais visam superar os obstáculos encontrados pela administração oral de fármacos, mais especificamente com relação ao desenvolvimento de sistemas terapêuticos dotados de elevado grau de sítio - alvo especificidade (Cavalcanti et al., 2002; Liu et al., 2003, Bunhak et al., 2007 a).

Dentre as justificativas para aplicação do revestimento polimérico das formas farmacêuticas sólidas orais, poderemos incluir: mascarar sabores e/ou odores desagradáveis, melhoria estética, proteção frente ao fluido gástrico, prevenção das interações entre constituintes da formulação, aliado ao aumento da estabilidade. Entretanto, a maior expectativa clínica, o maior grau de impacto proporcionado por este recurso tecnológico, recai sobre a possibilidade do efetivo controle da liberação temporal e espacial do fármaco. O processo de revestimento tem sido sugerido a partir da aplicação de materiais poliméricos reconhecidos como seguros, capazes de proporcionar liberação controlada e precisa do fármaco, e com excelente reprodutibilidade. Neste contexto destaca-se o desenvolvimento de novos materiais envolvendo polissacarídeos voltados à aplicação no controle da cinética de liberação de fármacos (Bunhak et al., $2007 a$; Sinha et al., 2003; Friend, 2005).
Diversos polissacarídeos não celulósicos das mais variadas origens: vegetais (ex.: pectina, goma arábica, goma guar, inulina e amilose); microorganismos (ex.: dextrana, goma xantana, ciclodextrina e pullulana), de alga (ex.: alginato) e de animais (ex.: sulfato de condroitina e quitosana) têm sido sugeridos como portadores de excelente potencial para aplicação no desenvolvimento de novos materiais destinados ao revestimento farmacêutico (Sinha et al., 2003; Friend, 2005).

Polímeros naturais apresentam grande disponibilidade na natureza, ampla variedade de estruturas, baixo custo de aquisição, alta estabilidade, boa propriedade geleificante, biocompatibilidade, atoxicidade e de fácil modificação química e bioquímica. Inúmeros oligopolissacarídeos têm sido propostos individualmente, modificados e/ou associados aos polímeros sintéticos, sendo estes últimos amplamente aplicados pela indústria farmacêutica (Bunhak et al., $2007 a, b$; Oliveira, Cavalcanti, 2007).

A pectina tem sido sugerida no desenvolvimento de sistemas terapêuticos colo-específicos, na formação de sistemas matriciais, reservatórios, além de combinada com outros polímeros (Dupuis et al., 2006). Encontrada em diversas espécies vegetais, principalmente nas células da parede celular, a pectina é responsável pela manutenção da estrutura e sustentação das plantas. Esse polissacarídeo apresenta-se predominantemente como um polímero linear constituído quimicamente por monômeros de ácido D-galacturônico em ligações $\alpha-(1-4)$, ocasionalmente interrompidos por monômeros de L-rhaminose em ligações $\alpha$-(1-2). Porém, outros monômeros também podem fazer parte das cadeias laterais, como D-galactose e L-arabinose, entre outros (Pérez et al., 2000; Pérez et al., 2003). Vale salientar que este polissacarídeo é resistente a passagem pelo segmento proximal do trato gastrintestinal, apresentando biodegradabilidade específica pela microflora anaeróbica, encontrando-se esta residente exclusivamente no colo (Sinha et al., 2001; Friend, 2005; Orlu et al, 2006).

A pectina apresenta elevado peso molecular, podendo variar entre 50.000 a $180.000 \mathrm{~g} / \mathrm{mol}$. Dependendo do grau de substituição dos grupos carboxila, presentes nos monômeros de ácido D-galacturônico, por grupos metoxila (-OCH3), a pectina pode ser classificada como de alta metoxilação / esterificação (acima de $50 \%$ ) e de baixa (abaixo de $50 \%$ ). Desse modo, a pectina pode ser descrita em termos de uma estrutura "canônica" por apresentar estrutura química bastante heterogênea e complexa. (Pérez et al., 2000; Pérez et al., 2003; Chourasia et al., 2003).

Alguns polissacarídeos naturais deparam com limitação de aplicação no desenvolvimento de novos materiais, princi- 
palmente quando destinados aos processos industriais para revestimento farmacêutico. Esta limitação tem sido representada prioritariamente pela alta solubilidade em meio aquoso, além da baixa propriedade filmogênica. Modificações estruturais dos polissacarídeos poderão contribuir para melhoria dessas limitações, evitando liberação prematura e indesejada do princípio ativo quando estes polímeros naturais estiverem inseridos como excipiente farmacotécnico em sólidos orais. Assim, apresenta-se como alternativa para reduzir a elevada solubilidade dos polissacarídeos, a modificação química, entretanto deve existir a preocupação da manutenção da expectativa de biodegradabilidade dos polissacarídeos pela microflora colônica (Sinha et al., 2003; Friend, 2005).

Associar polissacarídeos modificados aos polímeros sintéticos disponíveis no mercado em forma de dispersão aquosa, consagrados pela indústria farmacêutica, a exemplo do Surelease ${ }^{\circledR}$, Aquacoat $^{\circledR}, \mathrm{ECD}^{\circledR}$ e polímeros acrílicos, tais como Eudragit ${ }^{\circledR}$ NE 30 D, RS 30 D, RL 30 D e FS 30 D poderá melhorar a propriedade filmogênica dos polímeros naturais modificados. Esses polímeros sintéticos apresentam como maior vantagem a não utilização de solventes orgânicos em sua composição, os quais poderiam representar custos elevados do processo. Aliado a exigência de instalações industriais sofisticadas, dotadas de sistemas de exaustão arrojados com tratamento dos resíduos químicos produzidos, criando uma atmosfera de constante ameaça à saúde dos operadores, além de haver riscos de explosões e danos ao meio ambiente (Sinha et al., 2003, Ibekwe et al., 2006; Bunhak et al., 2007 a).

Essa associação polissacarídeo/polímero sintético sugere expectativa com relação à cinética de liberação e a sítio-especificidade do sistema, uma vez que a pectina deverá ser totalmente degradada pelas enzimas secretadas pela microflora presente na região colônica (principalmente Bacterióides, Bifidobacterium e Eubacterium) e o polímero sintético deverá garantir a integridade da película até as regiões distais (Chourasia et al., 2003).

Nesta investigação propusemos a formação e avaliação de filmes isolados gerados a partir da associação do polimetacrilato (Eudragit ${ }^{\circledR}$ RS $30 \mathrm{D}$ ) com o pré-biótico e com a pectina fosfatada (Pect-TMFT), a qual foi obtida através de reação de reticulação (meio aquoso baseificado) com trimetafosfato trisódico (TMFT) conforme representado na Figura 1. Essa reação gera material com solubilidade reduzida em meio aquoso, a exemplo de outros trabalhos já realizados com este mesmo agente reticulante. Esta diminuição de hidrofilicidade do polissacarídeo está relacionada à redução no número de hidroxilas livres para interagir com a água (Gliko-Kabir et al., 2000; Dulong et al., 2004; Lack et al., 2004; Cavalcanti et al., 2005; Braz, Hechenleitner, Cavalcanti, 2007).

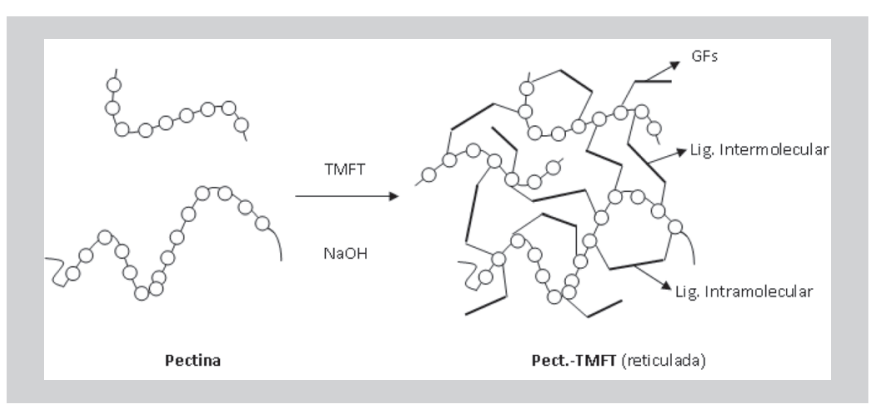

FIGURA 1 - Representação esquemática da reticulação da pectina. Enxerto de grupamentos fosfatos (GFs) na cadeia do polissacarídeo e formação de reticulação entre as cadeias poliméricas, com ligações intermoleculares e intramoleculares (adaptado de Franssen et al., 1999).

O pré-biotico $\alpha$-Gluco-oligossacarídeo (Bioecolians ${ }^{\circledR}$ ) agente responsável pela manutenção e equilíbrio do ecossistema intestinal também foi incorporado a esta formulação. Os prébioticos são substratos da microflora residente na região colônica, estando associados com a prevenção do câncer de colo, inibição do desenvolvimento de bactérias patogênicas, aumento da absorção de cálcio, melhoria da resposta imunológica além de contribuir na redução dos níveis de colesterol e triglicérides (Manning et al., 2004; Delzenne, 2003).

\section{MATERIAL E MÉTODOS}

\section{Material}

Pectina Genu ${ }^{\circledR}$ tipo USP-B (cedida gentilmente pela CPKelco, Limeira/SP), previamente modificada com trimetafofato trisódico (TMFT); Eudragit ${ }^{\circledR}$ RS 30 D (copolímero acrilato-metacrilato com grupos amônio quaternário USP/NF/Roehm Pharma/Alemanha) e citrato de trietila (TEC) (Morflex ${ }^{\circledR}$ - EUA) ambos cedidos gentilmente pela empresa Almapal (São Paulo/SP); Bioecolians ${ }^{\circledR}$ ( $\alpha$-Gluco-oligossacarídeo - França) cedido pela Solabia (Maringá/PR); Fluído de Simulação Gástrica (FSG, pH= 1,2) e Fluído de Simulação Intestinal (FSI, pH=6,8); Agitadores magnéticos (Tecnal ${ }^{\mathbb{R}}$ modelo: TE-085/1); Balança Analítica (Gehaka ${ }^{\circledR}$, modelo: AG200); Bomba de vácuo; Placa de Teflon ${ }^{\circledR}$; Micrômetro $\left(\right.$ Mitutoyo $\left.^{\circledR}\right)$; Tesoura cirúrgica (HVS cirúrgica ${ }^{\circledR}$, modelo: Íris); Estufa; Dessecadores (com sílica gel); Cúpulas de permeabilidade (Payne permiability cup, Braive Intriments, Liège, Bélgica); demais reagentes de grau analítico.

\section{Obtenção dos filmes com Pect-TMFT}

Os filmes foram confeccionados a partir de dispersões em base aquosa, utilizando método convencional para 
polímeros termoplásticos e termorrígidos, denominado evaporação, vazamento ou "casting process". Dispersões poliméricas foram preparadas em quatro diferentes concentrações, fazendo variar a concentração da Pect-TMFT (com 23\% m/m de TMFT) e/ou do pseudolatex (Eudragit ${ }^{\circledR}$ RS 30 D) e/ ou do $\alpha$-Gluco-oligossacarídeo $\left(\right.$ Bioecolians $\left.^{\circledR}\right)$, permanecendo sempre a massa polimérica final de $4 \%(\mathrm{p} / \mathrm{v})$. As proporções testadas foram (Eudragit ${ }^{\circledR}$ RS 30 D: Pect-TMFT: Bioecolians $^{\circledR}$ ) 90: 05: 05, 80: 10: 10, 70: 20: 10 e 100: 0: 0, corresponde à composição controle conforme Tabela I. O plastificante utilizado foi o citrato de trietila (TEC), adicionando na concentração de $20 \%$ em relação, exclusivamente, à massa do polimetacrilato (Eudragit ${ }^{\mathbb{R}} \mathrm{RS} 30 \mathrm{D}$ ).

As dispersões contendo inicialmente o Eudragit ${ }^{\circledR} \mathrm{RS}$ $30 \mathrm{D}$ acrescidas do TEC (20\%) foram deixadas sob agitação magnética por um período de 30 minutos em temperatura ambiente $\left(\mathrm{TA}=25^{\circ} \mathrm{C} \pm 2,0\right)$. Após completa homogeneização, seguindo as formulações sugeridas, quantidades variáveis das dispersões de Pect-TMFT e Bioecolians ${ }^{\circledR}$ foram adicionadas suavemente, sob agitação constante, às dispersões do polímero sintético, e mantidas sob agitação magnética por mais 60 minutos em TA. Durante todo o processo de homogeneização foi utilizada bomba de vácuo para evitar a incorporação de ar, formação de bolhas às misturas poliméricas finais que poderiam comprometer a integridade da película.

TABELA I - Proporção dos componentes dos filmes isolados obtidos (Eudragit ${ }^{\circledR}$ RS 30 D, Pect-TMFT e Bioecolians ${ }^{\circledR}$ ).

\begin{tabular}{lccc}
\hline $\begin{array}{l}\text { Eudragit }^{\circledR} \\
\text { RS 30 D }(\%)\end{array}$ & $\begin{array}{c}\text { Pect-TMFT } \\
(\%)\end{array}$ & $\begin{array}{c}\text { Bioecolians }^{\circledR} \\
(\%)\end{array}$ & $\begin{array}{c}\text { TEC } \\
(\%)\end{array}$ \\
\hline 100 & 0 & 0 & 20 \\
90 & 5 & 5 & 20 \\
80 & 10 & 10 & 20 \\
70 & 20 & 10 & 20 \\
\hline
\end{tabular}

TABELA II - Valores das médias das espessuras dos filmes obtidos (Eudragit ${ }^{\circledR}$ RS 30 D + Pect-TMFT (A e E) + Bioecolians $\left.^{\circledR}\right),(\mathrm{n}=5)$.

\begin{tabular}{lcc}
\hline Associações & Espessuras $(\mathrm{mm})$ & Desvio padrão \\
\hline 100: $00: 00$ & 0,0813 & $\pm 0,0126$ \\
90: $05: 05$ & 0,1213 & $\pm 0,0038$ \\
80: $10: 10$ & 0,1107 & $\pm 0,0092$ \\
70: $20: 10$ & 0,1727 & $\pm 0,0233$ \\
\hline
\end{tabular}

Após completa homogeneização das diferentes dispersões, amostras de $10 \mathrm{~mL}$, de cada dispersão, foram vertidas aos orifícios previamente demarcados sob placa de
Teflon ${ }^{\circledR}$. Esta placa foi então conduzida à estufa, nivelada e mantida a $50{ }^{\circ} \mathrm{C}$ (temperatura mínima para a formação dos filmes) por período de aproximadamente 15 horas até a formação dos filmes.

Os filmes obtidos foram retirados cuidadosamente do substrato e analisados quanto as suas características morfológicas macroscópicas (rachaduras, bolhas de ar ou quaisquer imperfeições que possam interferir nos ensaios posteriores). É de fundamental importância a verificação destas características, especialmente no tocante à presença de bolhas de ar e/ou rachaduras, uma vez que a integridade desses filmes garantirá a credibilidade e execução dos ensaios propostos. As espessuras dos filmes foram determinadas a partir de 5 pontos aleatórios da superfície para cada película, utilizando um Micrômetro (Mitutoyo ${ }^{\circledR}$ ) conforme a Tabela II. Em seguida, os filmes isolados selecionados foram armazenados individualmente, separados por papel impermeável, em dessecador (contendo sílica gel) até o momento dos ensaios (Cavalcanti et al., 2002; Codagnone et al., 2004; Oliveira, Cavalcanti, 2007; Bunhak et al., $2007 a, b)$.

\section{Estudo de Permeabilidade / Transmissão de Vapor d'água (TVA)}

A determinação da transmissão de vapor d'água constitui método simples e de fácil execução para avaliar in vitro a permeabilidade através de filmes poliméricos quando comparado aos tradicionais métodos de difusão. É capaz de fornecer informação valiosa sobre a capacidade de proteção de diversos materiais (papéis, plástico, embalagens) contra a umidade do meio ambiente, inclusive dos materiais poliméricos envolvidos na tecnologia de revestimento farmacêutico durante a estocagem (Akhgari et al., 2006; Bunhak et al., 2007 a). Este estudo foi desenvolvido de acordo com o método "B" da ASTM (American Society for Testing and Material) designado E96-66, usando cúpulas de permeabilidade (modelo "Payne permeability cup", Bélgica). Os filmes candidatos à investigação (com área de $10 \mathrm{~cm}^{2}$ ) foram fixados às cúpulas individualmente, nas quais foram previamente preenchidas com água destilada $(10 \mathrm{~mL})$. O conjunto (cúpula + água destilada + amostra filme; $n=3$ ) foi pesado no tempo zero e armazenado em dessecador contendo sílica-gel desidratada. $\mathrm{O}$ dessecador foi deixado à temperatura e umidade ambiente. As cúpulas de cada filme foram pesadas (em triplicatas) nos intervalos de tempo: 0, 24, 48, 72, 96 e 120 horas. A cada intervalo de tempo pré-estabelecido, a amostra de sílica-gel foi trocada por outra desidratada $\left(110^{\circ} \mathrm{C} /\right.$ $1 \mathrm{~h})$. Os pesos perdidos pelas cúpulas nos respectivos intervalos de tempo foram registrados e aplicados na Equa- 
ção (1), sendo esta utilizada para calcular a taxa de transmissão de vapor d'água transportada através dos filmes. A TVA foi padronizada para um período de $24 \mathrm{~h}$.

$\mathrm{TVA}=\mathrm{g} \cdot 24 / \mathrm{t} \cdot \mathrm{a}$

Onde "g" é a perda de massa, " $t$ " é o tempo medido em horas onde houve perda de massa e "a" representa a área do filme $\left(0,001 \mathrm{~m}^{2}\right)$. Posteriormente, os valores percentuais das perdas dos pesos foram graficamente plotados versus os respectivos intervalos de tempo (Cavalcanti et al., 2002; Bunhak et al., 2006; Cavalcanti et al., 2005; Oliveira, Cavalcanti, 2007; Lamin et al., 2006).

\section{Determinação do Índice de Intumescimento (I \%)}

Os filmes nas suas variadas associações foram minuciosamente cortados com tesoura cirúrgica (modelo Professional F/1) em aproximadamente $1,0 \mathrm{~cm}^{2}$, sendo, em seguida, distribuídos no interior de placas de Petri devidamente identificadas.

Posteriormente, as placas de Petri foram colocadas em estufa a $50^{\circ} \mathrm{C}$, por aproximadamente 15 horas, atingindo perda total da umidade. Após este tempo, as placas foram retiradas e mantidas em dessecadores (com sílica gel) durante a realização do experimento propriamente dito.

As amostras secas das diferentes associações foram pesadas em balança analítica e imediatamente imersas em recipientes contendo Fluído de Simulação Gástrica (FSG, $\mathrm{pH}=1,2$ ) e Fluído de Simulação Intestinal (FSI, $\mathrm{pH}=6,8$ ), sem a presença de enzimas digestivas, e mantidas por diferentes intervalos de tempo à temperatura de $37^{\circ} \mathrm{C}$. Esses fluídos foram realizados segundo a $28^{\mathrm{a}}$ Farmacopéia dos Estados Unidos (USP XXVIII).

Cumpridos os intervalos de tempo pré-estabelecidos (1, 10, 30 e 60 minutos de imersão), as amostras foram removidas dos meios de simulação com auxílio de pinça e cuidadosamente enxugadas entre duas folhas de papel de filtro e repesadas. $\mathrm{O}$ mesmo procedimento foi adotado para todas as outras amostras em triplicatas.

Para o cálculo do Índice de Intumescimento ( $\left.\mathrm{I}_{\mathrm{i}} \%\right)$ foi utilizada a equação:

$I_{i} \%=\frac{(M i-M s) \times 100}{M s}$

Onde "Mi" é a massa da membrana após o intumescimento e "Ms" é a massa da membrana seca (Cavalcanti et al., 2002; Codagnone et al., 2004; Bunhak et al., 2007 a, b, Oliveira, Cavalcanti, 2007; Akhgari et al., 2006).

\section{Microscopia Eletrônica de Varredura (MEV)}

Amostras dos filmes isolados, secos e/ou imersos em FSG $(\mathrm{pH}=1,2)$ e FSI $(\mathrm{pH}=6,8)$, foram congeladas com nitrogênio líquido após realização do ensaio de $\mathrm{I}_{\mathrm{i}} \% \mathrm{e}$ liofilizadas a $-55^{\circ} \mathrm{C}$ (Liofilizador Martin Christ ${ }^{\circledR}$, Alpha 11/DL) por 6 horas na tentativa de preservar suas características morfológicas em ambas as condições.

Micrografias dos filmes isolados foram obtidas através do Microscópio Eletrônico de Varredura (Shimadzu modelo SS-550 - Superscan), operado em $12 \mathrm{keV}$. Todas as micrografias foram obtidas das superfícies de fratura recobertas com ouro.

\section{Análise estatística}

Análises estatísticas foram usadas para determinar o nível de significância existente entre os valores obtidos em ensaios de determinação do $\mathrm{I}_{\mathrm{i}} \%$, assim como da permeabilidade (TVA) para as diferentes associações, envolvendo os filmes com Pect-TMFT. Estas análises foram realizadas com ajuda do programa GraphPad Prism ${ }^{\circledR}$ (versão 2.01, 1996). Os diferentes resultados referentes aos coeficientes de TVA e $\mathrm{I}_{\mathrm{i}} \%$, obtidos nas diferentes circunstâncias (FSG e FSI), foram inicialmente avaliados usando análise de variância (ANOVA), sendo os resultados considerados significativos quando $\mathrm{p}<0,05$. Quando o conjunto desses valores forneceu parâmetros significativos, realizamos a aplicação das médias dos dados obtidos no teste de múltipla comparação de Tukey, visando comparar o papel das diferentes composições poliméricas. Também foram considerados significativos os valores registrados para $\mathrm{p}<0,05$.

\section{RESULTADOS E DISCUSSÃO}

\section{Características macroscópicas dos filmes}

Na Tabela III encontram-se os resultados das características mais evidentes nas composições poliméricas propostas. Os filmes apresentaram características macroscópicas referentes à transparência e flexibilidade adequadas, influenciadas e proporcionalmente dependentes da concentração do polissacarídeo modificado (PectTMFT). A presença do plastificante (20\%), favoreceu a coalescência do Eudragit ${ }^{\circledR}$ RS $30 \mathrm{D}$, associado à PectTMFT e ao Bioecolians ${ }^{\circledR}$, nas associações aplicadas (90: 05: 05, 80: 10: 10 e 70: 20: 10). Segundo Petereit e Weisbrod (1999) e Aydinli e Tutas (2000) essa melhor coalescência é atribuída à capacidade dos plastificantes em reduzir a temperatura de transição vítrea $\left(\mathrm{T}_{\mathrm{g}}\right)$ dos polímeros a eles associados, o que aumenta, como conseqüência, a 
Tabela III - Principais características macroscópicas observadas nas diferentes composições (Eudragit ${ }^{\circledR}$ RS $30 \mathrm{D}+$ Pect$\operatorname{TMFT}\left(\mathrm{A}\right.$ e E) + Bioecolians $\left.{ }^{\circledR}\right)$

\begin{tabular}{lccccc}
\hline $\begin{array}{l}\text { Composição } \\
\left(\begin{array}{l}\text { Eudragit }^{\circledR} \text { RS 30 D }+ \\
\text { Pect-TMFT + Bioecolians }^{\circledR} \text { ) }\end{array}\right.\end{array}$ & $\begin{array}{c}\text { Separaçãode } \\
\text { Fase }\end{array}$ & $\begin{array}{c}\text { Presençade } \\
\text { Rachadura }\end{array}$ & Bolha de Ar & Transparência & Flexibilidade \\
\hline 100:00:00 & 0 & 0 & 0 & +++ & +++ \\
90:05:05 & 0 & 0 & 0 & ++ & +++ \\
80:10:10 & 0 & 0 & 0 & ++ & ++ \\
$70: 20: 10$ & 0 & 0 & 0 & + & ++ \\
\hline
\end{tabular}

0: Não observada alteração; +: Levemente presente; ++: Mediamente presente; +++: Fortemente presente.

mobilidade das cadeias poliméricas, melhorando a propriedade mecânica dos filmes.

Interessante observar, conforme a Tabela II, que o aumento das espessuras dos demais filmes em relação ao filme padrão (Eudragit ${ }^{\mathbb{R}}$ RS 30 D 100: 00: 00) está relacionado ao aumento da concentração de Pect-TMFT. Trabalhos anteriormente realizados por Gabas e Cavalcanti (2003) e Bunhak et al. $(2007$, b) também observaram situação semelhante, onde o polissacarídeo modificado gerou aumento nas espessuras dos filmes isolados formados.

\section{Estudo de Permeabilidade / Transmissão de Vapor d'água (TVA)}

Os resultados registrados na Tabela IV demonstram que a taxa de TVA sofre influência conforme a composição do filme quando comparados entre si, incluindo o controle 100: 00: 00 do polimetacrilato. $\mathrm{O}$ aumento na concentração de PectTMFT e do pré-biótico gerou aumento na taxa de transmissão de vapor d'água, caracterizada pela perda de peso do dispositivo (Tabela IV). Este fenômeno pode ser justificado pelo aumento da hidrofilicidade do sistema causado pelo aumento no número de hidroxilas disponíveis para interagir com moléculas de água. No entanto, o conjunto desses valores não apresentou resultados estatisticamente significativos $(p<0,05)$, segundo análise de variância (ANOVA).

Rosina e colaboradores (2004), quando produziram filmes isolados de Eudragit ${ }^{\mathbb{B}} \mathrm{RS} 30 \mathrm{D}$ associados ao polímero extraído da raiz de Nelumbo nucifera, constataram um aumento da taxa de transmissão de vapor d'água proporcional e dependente do aumento da concentração do polissacarídeo incorporado. Cavalcanti e colaboradores (2002), utilizando o Eudragit ${ }^{\mathbb{B}}$ RS $30 \mathrm{D}$ associado à galactomanana ou à inulina, também observaram semelhante comportamento na permeabilidade, proporcional ao aumento da concentração do oligo-polissacarídeo. Esta mesma tendência de aumento da transmissão de vapor d'água pode ser observada na Tabela IV e Figura 2 para as composições envolvendo a pectina fosfatada (Pect-TMFT) e Bioecolians ${ }^{\circledR}$ nas concentrações 95: 05: 05, 80: 10: 10 e 70: 20: 10, quando comparados ao padrão (100:00:00 Eudragit ${ }^{\circledR}$ RS 30 D).

Tabela IV - Valores de taxa de Transmissão de Vapor d'água e perda de peso do controle (100: 00: 00, Eudragit ${ }^{\mathbb{B}}$ RS 30 D) e das associações 90: 05: 05, 80:10: 10 e 70: 20: $10\left(\right.$ RS 30 D + Pect-TMFT + Bioecolians ${ }^{\circledR},(n=3)$.

\begin{tabular}{lcc}
\hline $\begin{array}{l}\text { Associação } \\
\text { polimérica }\end{array}$ & $\begin{array}{c}\text { TVA } \\
\left(\mathrm{g} / \mathrm{m}^{2} 24 \mathrm{~h}\right)\end{array}$ & $\begin{array}{c}\text { Valores perda de } \\
\text { peso }(\mathrm{g} / 120 \mathrm{~h})\end{array}$ \\
\hline 100: 00: 00 & $416,900( \pm 18,142)$ & $2,086( \pm 0,134)$ \\
90: $05: 05$ & $517,400( \pm 14,315)$ & $2,449( \pm 0,025)$ \\
80: $10: 10$ & $754,467( \pm 21,607)$ & $3,435( \pm 0,075)$ \\
70: $20: 10$ & $861,267( \pm 11,136)$ & $3,742( \pm 0,062)$ \\
\hline
\end{tabular}

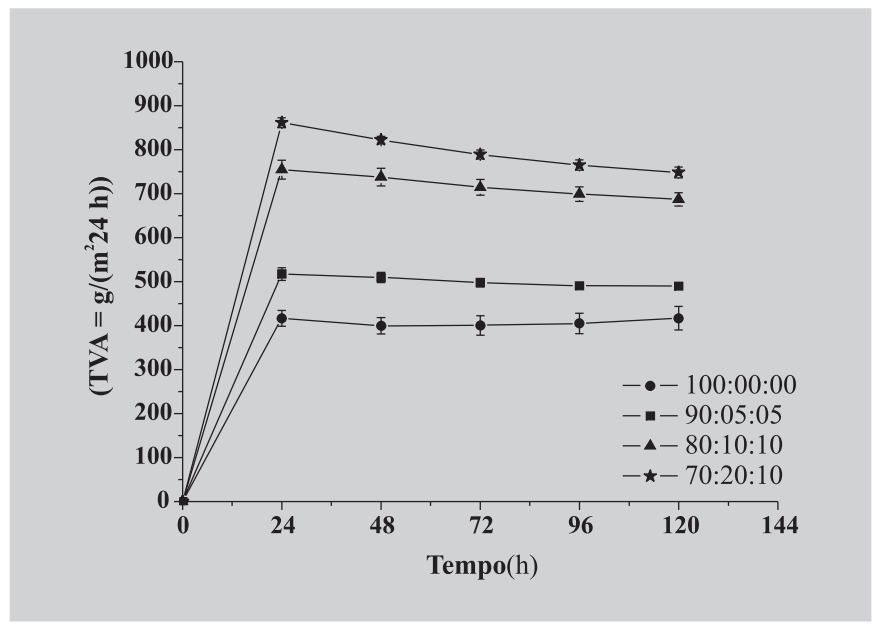

FIGURA 2 - Transmissão de Vapor d'água (TVA $=\mathrm{g} /\left(\mathrm{m}^{2}\right.$ 24 h)) do Controle (100: 00: 00, Eudragit ${ }^{\circledR}$ RS 30 D e das associações 90: 05: 05, 80: 10: 10 e 70: 20: 10 (Eudragit $^{\circledR}$ RS 30 D + Pect-TMFT + Bioecolians ${ }^{\circledR},(n=3)$. 
Esta característica de alteração da permeabilidade desperta grande interesse, e encontra-se vinculada ao aumento da concentração do polissacarídeo modificado e a adição do pré-biótico em sua composição. Esta combinação de materiais provavelmente proporcionará material filmogênico detentor de maior vulnerabilidade à fermentação específica no ambiente colônico e poderá aperfeiçoar o controle na difusão de fluidos e fármacos.

\section{Determinação do Índice de Intumescimento $\underset{i}{(\mathrm{i} \%)}$}

Conforme citado por Oliveira e Cavalcanti (2007), o conhecimento do grau de hidrofilicidade dos filmes isolados quando em contato com os fluidos fisiológicos constitui uma característica imprescindível, fenômeno que deverá garantir o acesso das enzimas bacterianas existentes na porção distal do TGI. Além disso, a avaliação do $\left(\mathrm{I}_{\mathrm{i}} \%\right)$ pode representar o primeiro passo para a elaboração de um modelo matemático capaz de prever a cinética de liberação (Mulhbacher et al., 2004).

O índice de intumescimento foi aplicado para determinar o grau de hidratação dos filmes isolados obtidos conforme as Figuras 3 e 4 . A análise estatística (ANOVA) sobre o conjunto dos dados gerados nos experimentos realizados, registrou diferenças significativas $(\mathrm{p}<0,05)$ entre as composições quando comparadas ao controle (100:00:00, Eudragit $\left.^{\mathbb{B}} \mathrm{RS} 30 \mathrm{D}\right)$. Entretanto, quando o conjunto desses valores foi submetido ao teste de múltipla comparação de Tukey, apenas as composições 90: 05: 05 versus 70: 20: 10 apresentaram parâmetros significativos $(\mathrm{p}<0,05)$.

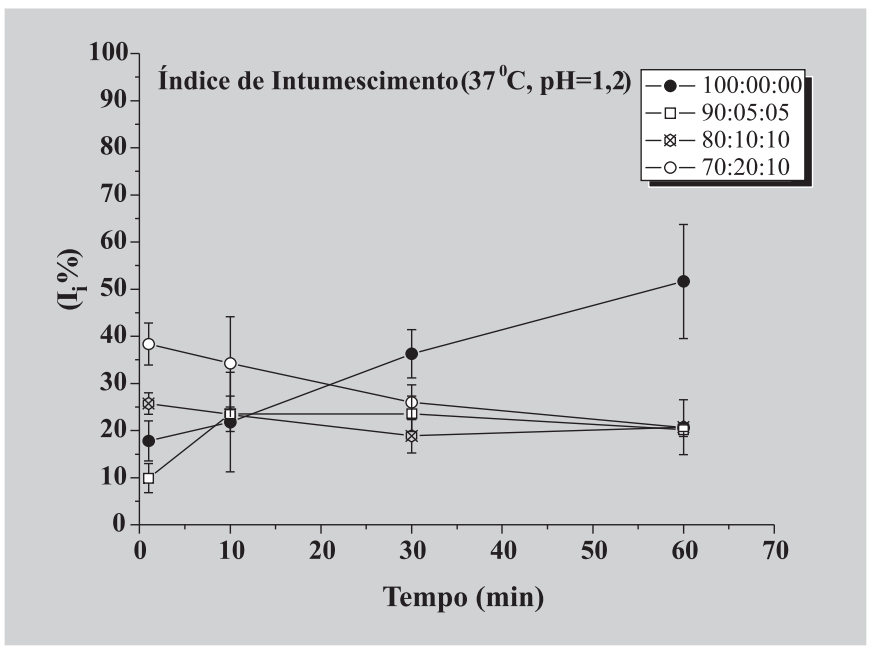

FIGURA 3 - Representação gráfica do índice de intumescimento. Avaliação da hidratação do filme padrão (100: 00: 00, Eudragit ${ }^{\circledR}$ RS 30 D) e das associações 90: 05: 05, 80: 10: 10 e 70: 20: 10 (RS 30 D + Pect-TMFT + Bioecolians $\left.{ }^{\circledR}\right)$ em FSG $(\mathrm{pH}=1,2),(\mathrm{n}=3)$.
Conforme observado na Figura 3, em FSG a hidratação dos filmes contendo pectina reticulada com TMFT foi menor devido ao $\mathrm{pH}$ ácido, o que causa um maior entrelaçamento da malha polimérica, diminuindo conseqüentemente, a retenção de água. De modo contrário conforme a Figura 4, em FSI o pH aumentado causou ionização dos grupos carboxilatos e, conseqüentemente, uma expansão da malha polimérica, ou seja, maior hidratação (Gliko-kabir et al., 2000).

Observando-se a Figura 4, é possível constatar que os valores de hidratação dos filmes quando em FSI, sofreram influência da concentração do polissacarídeo modificado. Isto interfere de forma significativa na hidratação do polissacarídeo fosfatado, o qual possui uma característica fortemente aniônica devido à presença de grupos carboxilatos e grupos fosfatos. Segundo comenta Mulhbacher et al. (2004), com o aumento do $\mathrm{pH}$ espera-se que o intumescimento de polímeros aniônicos, a exemplo da pectina fosfatada, aumente.

Esta constatação também foi observada por GlikoKabir et al. (2000), quando avaliando o grau de hidratação da goma guar fosfatada em fluidos FSG e FSI, evidenciou este mesmo perfil de comportamento.

No FSI $(\mathrm{pH}=6,8)$ representado pela Figura 4, foi possível observar nos primeiros 10 minutos um intumescimento acentuado para as composições com $20 \%$ de Pect-TMFT (70: 20: 10) seguido de uma saturação após 10 minutos. Após este período (10 minutos) constatamos uma diminuição do $\mathrm{I}_{\mathrm{i}} \%$, este comportamento poderá ser atribuído à migração da pectina (Pect-TMFT) e/ou Bioecolians ${ }^{\circledR}$ ao meio. Resultado

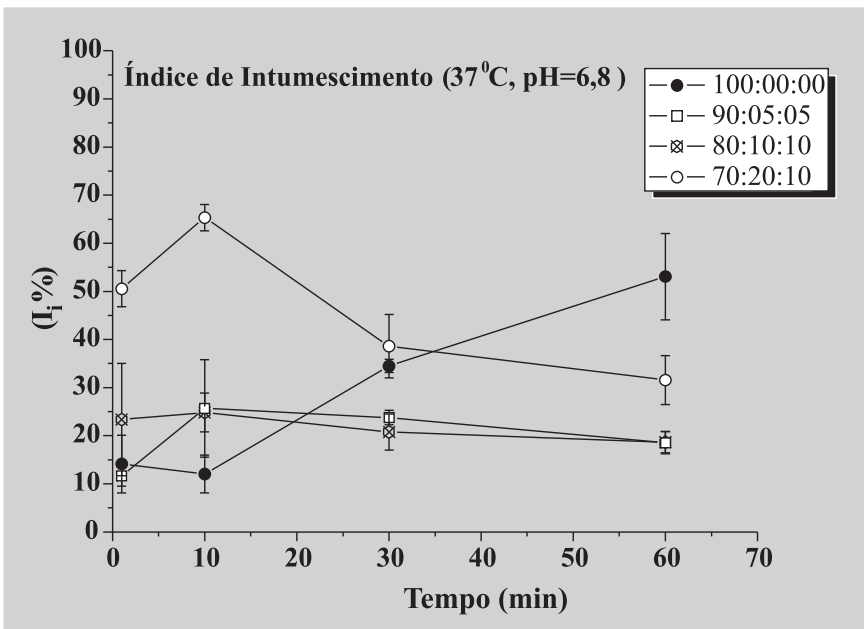

FIGURA 4 - Representação gráfica do índice de intumescimento. Avaliação da hidratação do filme padrão (100: 00: 00, Eudragit ${ }^{\circledR}$ RS 30 D) e das associações 90: 05: 05, 80: 10: 10 e 70: 20: 10 (RS 30 D + Pect-TMFT + Bioecolians $\left.^{\circledR}\right)$ em FSI $(\mathrm{pH}=6,8),(\mathrm{n}=3)$. 
bastante semelhante foi observado por Semdé e colaboradores (1998) durante avaliação de filmes contendo Eudragit ${ }^{\mathbb{R}} \mathrm{RS}$ $30 \mathrm{D}$ e pectinato de cálcio.

A formação de grupos carboxilatos ocorrida com a reação de fosfatação da pectina provavelmente impede a interação dos grupos aniônicos (ácidos carboxílicos) da pectina com os grupos catiônicos (amônio quaternário) do Eudragit ${ }^{\circledR}$ RS 30 D. Assim, quando em FSI $(\mathrm{pH}=6,8)$, devido ao maior relaxamento da malha polimérica, podese sugerir que a pectina fosfatada e o Bioecolians ${ }^{\circledR}$ poderão ser liberados para o meio por não formar complexo com o polímero de base.

Oliveira e Cavalcanti (2007) comparando o comportamento de hidratação do controle (100:00:00, Eudragit ${ }^{\circledR}$ RS 30 D) em FSG e FSI não observaram diferenças significativas. Nossos resultados apresentados nas Figuras 3 e 4 estão de acordo com as observações desses autores, já que esse copolímero (de ésteres de ácido acrílico e metacrílico) é insolúvel em água, porém possui grupos amônios quaternários responsáveis pelo intumescimento lento do polímero na presença de fluídos digestivos independentemente do $\mathrm{pH}$ do meio.

\section{Microscopia Eletrônica de Varredura (MEV)}

Observando-se as micrografias obtidas das amostras dos filmes após realização do ensaio de $\mathrm{I}_{\mathrm{i}}$ \% FSG e FSI, conforme a Figura 5 foi possível observar mudanças em suas características morfológicas quando comparadas com a amostra do filme seco (Figura 5, A e D).

Quando submetido ao FSG $(\mathrm{pH}=1,2)$ foi possível observar uma superfície bastante heterogênea com a presença marcante da Pect-TMFT e do Bioecolians ${ }^{\circledR}$ (Figura 5 , B e E). De modo contrário, quando as películas foram imersas em FSI $(\mathrm{pH}=6,8)$, as amostras dos filmes isolados apresentaram maior quantidade de poros em determinadas regiões (Figura 5, C e F), estando estes provavelmente relacionados à migração da Pect-TMFT e do Bioecolians ${ }^{\circledR}$ para o meio, conforme anteriormente discutido na determinação do índice de intumescimento, fenômeno relacionado ao maior relaxamento da malha polimérica quando em $\mathrm{pH}$ $=6,8$, e por não formar complexo com o polímero de base.

\section{CONCLUSÃO}

Os resultados obtidos nesta investigação demonstraram que a adição do oligossacarídeo e polissacarídeo (reticulado) ao polimetacrilato Eudragit ${ }^{\circledR}$ RS 30 D em dispersão aquosa promoveu alterações na propriedade de permeabilidade e de hidratação dos filmes formados, quando comparados ao controle (100: 00: 00, Eudragit ${ }^{\circledR}$ RS 30 D), sendo estas dependentes e proporcionais ao aumento da concentração da pectina fosfatada (Pect-TMFT) e do prébiótico (Bioecolians ${ }^{\circledR}$ ) inclusos nos filmes.

O filme obtido e avaliado nessa etapa, na concentração 70: 20: 10 (Pect-TMFT + Eudragit ${ }^{\circledR}$ RS 30 D + Bioecolians $^{\circledR}$ ) apesar de ter apresentado um comportamento anômalo durante o ensaio de $\mathrm{I}_{\mathrm{i}} \%$ quando submetido ao

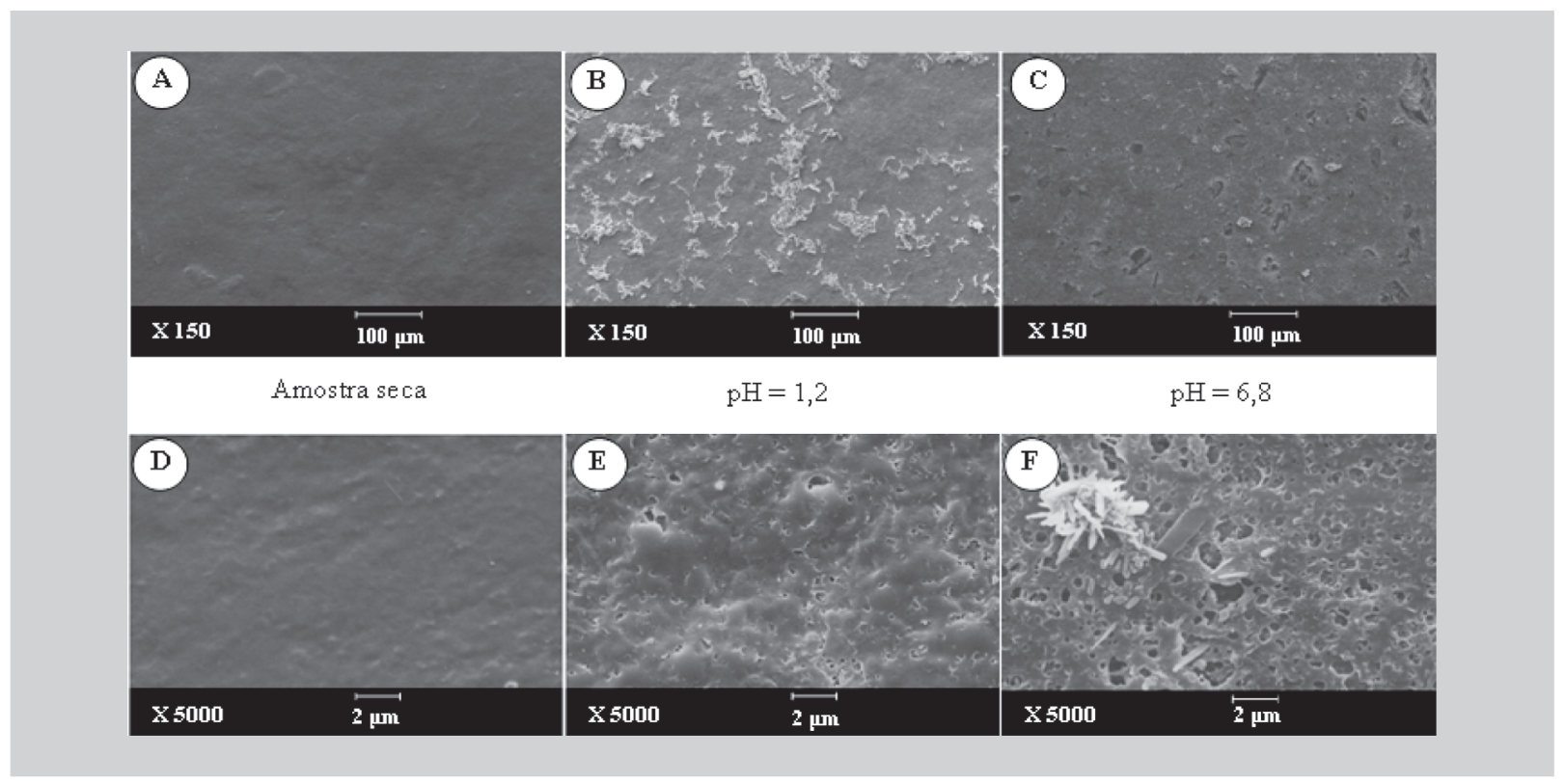

FIGURA 5 - Micrografias obtidas por meio de MEV do filme de Eudragit ${ }^{\circledR}$ RS 30 D: Pect-TMFT: Bioecolians ${ }^{\circledR}, 70: 20$ : 10. $\operatorname{Em}(A)$ e (D): amostra seca; (B) e (E): $\mathrm{pH}=1,2$ e (C) e (F): $\mathrm{pH}=6,8$. 
FSI $(\mathrm{pH}=6,8)$, poderá impedir a liberação prematura do fármaco em região proximal do TGI quando utilizado para revestimento de sistemas sólidos orais.

Além disso, as presenças da pectina fosfatada e do Bioecolians ${ }^{\circledR}$ poderão possibilitar uma degradação específica da película por enzimas produzidas pela microflora colônica, independente de variações de $\mathrm{pH}$ que possam existir entre diferentes indivíduos, possibilitando uma cinética de liberação modificada de fármacos sítio-alvo-específica. Porém, ensaios posteriores de colo-especificidade in vitro e/ou vivo, poderão confirmar a aplicação deste novo material.

\section{ABSTRACT \\ Phosphated pectin application in the development of films for drug delivery systems. Evaluation of permeability properties and swelling}

Phosphated pectin (Pect-TMFT) together with $\alpha$-glucooligossacaride (Bioecolians ${ }^{\circledR}$ ) were incorporated into aqueous dispersion of polymethacrylate (Eudragit ${ }^{\circledR} R S 30$ D) to obtain free films by the casting process $\left(50^{\circ} \mathrm{C}\right)$ in Teflon ${ }^{\circledR}$ plate. Pect-TMFT and Bioecolians ${ }^{\circledR}$ were added into dispersions of Eudragit ${ }^{\circledR} R S 30 \mathrm{D}$ at different rates: 90:05:05, 80:10:10 and 70:20:10 (4\% p/v). Triethyl citrate (TEC) was used as plasticizer (20\% of mass of the polymethacrylate). The proposed dispersions showed film formation ability. The free films were characterized by the determination of water vapour transmission (WVT), by the swelling index ( $\left.I_{i} \%\right)$ in fluids of gastric simulation (FGS) and intestinal (FIS) and by scanning electron microscopy (SEM). The increase of modified polysaccharide and Bioecolians ${ }^{\circledR}$ in the films favored their permeability to the water vapour and their hydration degree when in FIS ( $\mathrm{pH}$ $=6.8$ ). In that way, the obtained film in the concentration 70:20:10, can prevent the premature release of the drug in the up GIT when applied to develop oral solid systems coating. Besides, the presence of Pect-TMFT and Bioecolians ${ }^{\circledR}$ can contribute to the specific membrane degradation by colonic microflora enzymes, making possible a modified release kinetics of drugs even with the existence of inter-individual variations of $\mathrm{pH}$.

UNITERMOS: Phosphated pectin. Free films. $\alpha$-Glucoolygosaccharides. Eudragit ${ }^{\circledR} R S 30$ D. Drugs/Modified release.

\section{AGRADECIMENTOS}

À empresa CPKelco (Limeira/SP) pela amostra de pectina USP tipo-B. À empresa Almapal (São Paulo/SP) pelas amostras dos produtos Eudragit ${ }^{\circledR}$ RS 30 D - Degussa/ Alemanha, e Citrato de trietila (Morflex ${ }^{\circledR}-$ EUA). E à empresa Solabia (Maringá/PR) pela amostra de Bioecolians ${ }^{\circledR}$. Projeto Institucional PPG-UEM, processo 144/2006.

\section{REFERÊNCIAS BIBLIOGRÁFICAS}

AHMED, A.; BONNER, C.; DESAI, T. A. Bioadhesive microdevices with multiple reservoirs: a new platform for oral drug delivery. J. Control. Release, Amsterdam, v.81, n.3, p.291-306, 2002.

AKHGARI, A.; FARAHMAND, F.; GAREKANI, H. A.; SADEGHI, F.; VANDAMME, T. F. Permeability and swelling studies on free films containing inulin in combination with different polymethacrylates aimed for colonic drug delivery. Eur. J. Pharm. Sci., Amsterdam, v.28, n.4, p.307-314, 2006.

AYDINLI, M.; TUTAS, M. Water Sorption and Water Vapour Permeability Properties of Polysaccharide(Locust Bean Gum) Based Edible Films. Lebensm-Wiss. Technol., Amsterdam, v.33, n.1, p.63-67, 2000.

BRAZ, R.; HECHENLEITNER. A. A. W.; CAVALCANTI, O. A. Extraction, structural modification and characterization of Lotus roots polysaccharides (Nelumbo nucifera Gaertn). Excipient with potential application in modified drug delivery systems. Lat. Am. J. Pharm., La Plata, v.26, n.5, p.706-710, 2007.

BUNHAK, E. J.; MENDES, E. S.; PEREIRA, N. C.; CAVALCANTI, O. A. Influência do Sulfato de Condroitina na Formação de Filmes Isolados de Polimetacrilato: Avaliação do Índice de Intumescimento e Permeabilidade ao Vapor d'água. Quím. Nova, São Paulo, v.30, n.2, p.312-317, 2007,a.

BUNHAK, E. J.; MENDES, E. S.; PEREIRA, N. C.; CAVALCANTI, O. A. Influência do Sulfato de Condroitina na Formação de Filmes Isolados de Etilcelulose. Avaliação das Características de Hidratação e Permeabilidade. Lat. Am. J. Pharm., La Plata, v.26, n.1, p.89-95, 2007b.

CAVALCANTI, O.A.; MOOTER, G. V. Den; CARAMICOSOARES, I.; KINGET, R. Polysaccharides as Excipients for Colon-Specific Coatings. Permeability and Swelling Properties of Casted Films. Drug Dev. Ind. Pharm., New York, v.28, n.2, p.157-164, 2002. 
CAVALCANTI, O. A.; SILVA, C. C. da; PINEDA, E. A. G.; HECHENLEITNER, A. A. W. Synthesis and Characterization of Phosphated Crosslinked Chondroitin Sulfate: Potential Ingredient for Specific Drug Delevery. Acta Farm. Bonaerense, La Plata, v. 24, n.2, p.1-5, 2005.

CHOURASIA, M. K.; JAIN, S. K. Pharmaceutical approaches to colon targeted drug delivery systems. $J$. Pharm. Sci., Amsterdam, v.6, n.1, p.33-66, 2003.

CODAGNONE, A. F.; HECHENLEITNER, A. A. W.; PINEDA, E. A. G.; CAVALCANTI, O. A. Goma Guar Fosfatada: Potencial Excipiente no Desenvolvimento de Filmes Isolados de Etilcelulose. Acta Farm. Bonaerense, La Plata, v.23, n.4, p.448-452, 2004.

DELZENNE, N. M. Oligosaccharides: state of the art. P. Nutr. Soc., Hyderabad, v.62, n.1, p.177-182, 2003.

DULONG, V.; LACK, S.; CERF, D. Le; PICTON, L.; VANNIER, J. P.; MULLER, G. Hyaluronan-based hydrogels particles prepared by crosslinkingwith trisodium trimetaphosphate. Synthesis and characterization. Carbohyd. Polym., Oxford, v.57, n.1, p.1-6, 2004.

DUPUIS, G.; CHAMBIN, O.; GENELOT, C.; CHAMPION, D. and POURCELOT, Y. Colonic Drug delivery: Influence of cross-linking Agent on Pectin Beads properties and Role of the Shell Capsule type. Drug Dev. Ind. Pharm., New York, v.32, n.7, p.847-855, 2006.

FRANSSEN, O.; OOIJEN, R. D. van; BOER, D. de; MAES, R. A. A.; HENNINK, W. E. Enzymatic Degradation of Cross-Linked Dextrans. Macromolecules, London, v.32, n.9, p.2896-2902, 1999.

FRIEND, D. R. New oral delivery systems for treatment of inflammatory bowel disease. Adv. Drug Deliver. Rev., Amsterdam, v.57, n.2, p.247-265, 2005.

GABAS, V. G. S.; CAVALCANTI, O. A. Influência da adiçabo da goma araibica em filmes isolados de polìmero acriilico. Estudo das propriedades de intumescimento e de permeabilidade. Rev. Bras. Cien. Farm., São Paulo, v.39, n.4, p.441-448, 2003.
GLIKO-KABIR, I.; YAGEN, B.; PENHASI, A.; RUBINSTEIN, A. Phosphated crosslinked guar for colon-specific drug delivery. I. Preparation and physicochemical characterization. J. Control. Release, Amsterdan, v.63, n.1-2, p.121-127, 2000.

IBEKWE, V. C.; FADDA, H. M.; PARSONS, G. E.; BASIT, A. W. A comparative in vitro assessment of the drug release performance of $\mathrm{pH}$-responsive polymers for ileocolonic delivery. Int. J. Pharm., Amsterdam, v.308, p.5260, 2006.

IRACHE, J. M., HUICI, M., KONECNY, M., ESPUELAS, S., CAMPANERO, M. A.,e ARBOS, P. Bioadhesive Properties of Gantrez Nanoparticles. Molecules, Basel, v.10, n.1, p.126-145, 2005.

LACK, S.; DULONG, V.; CERF, D. Le; PICTON, L.; ARGILLIER, J. F.; MULLER, G. Hydrogels Based on Pullulan Crosslinked with sodium trimetaphosphate (STMP): Rheological study. Polym. Bull., Heidelberg, v.52, p.429-436, 2004.

LAMIM, R.; FREITAS, R. A. de; RUDEK, E. I.; WILHELM, H. M.; CAVALCANTI, O. A.; BRESOLIN, T. M. B. Films of chitosan and $N$-carboxymethylchitosan. Part II: Effect of plasticizers on their physiochemical properties. Polym. Int., London, v.55, n.8, p.970-977, 2006.

LIU, L.; FISHMANA, M. L.; KOSTB, J.; HICKS, K. B. Pectin-based systems for colon-specific drug delivery via oral route. Biomaterials, Amsterdam, v.24, n.19, p.3333$3343,2003$.

MANNING, T.S.; GIBSON, G. R. Prebiotics. Best Pract. Res. Cl. Ga., Amsterdam, v,18, n.2, p.287-298, 2004.

MASTIHOLIMATH, V. S.; DANDAGI, P. M.; JAIN, S. S.; GADAD, A. P.; KULKARNI, A. R. Time and $\mathrm{pH}$ dependent colon specific, pulsatile delivery of theophylline for nocturnal asthma. Int. J. Pharm., Amsterdam, v.328, n.1, p.49-56, 2007.

OLIVEIRA, F. M.; CAVALCANTI, O. A. Pré-Bióticos na Formação de filmes isolados de Eudragit ${ }^{\circledR}$ RS30D. Avaliação das propriedades de intumescimento e permeabilidade. Lat. Am. J. Pharm., La Plata, v.26, n.3, p.325-31, 2007. 
ORLU, M.; CEVHER, E.; ARAMAN, A. Design and evaluation of colon specific drug delivery system containing flurbiprofen microsponges. Int. J. Pharm., Amsterdam, v.318, n.1-2, p.103-117, 2006.

PÉREZ, S.; MAZEAU, K.; PENHOAT, C. H. du. The threedimensional structures of the pectic polysaccharides. Plant Physiol. Bioch., Amsterdam, v.38, n.1-2, p.37-55, 2000.

PÉREZ, S.; RODRÍGUEZ-CARVAJAL, M. A.; DOCO, T. A complex plant cell wall polysaccharide: rhamnogalacturonan II. A structure in quest of a function. Biochimie, Amsterdam, v.85, n.1-2, p.109-121, 2003.

PETEREIT, Hans-Ulrich; WEISBROD, W. Formulation and process considerations affecting the stability of solid dosage forms formulated with methacrylate copolymers. Eur. J. Pharm. Biopharm., Amsterdam, v.47, n.1, p.1525, 1999.
ROSINA, C. R.; BARONI, S.; CAVALCANTI, O. A. Avaliação das propriedades de intumescimento e permeabilidade de filmes isolados de polimetacrilato contendo polissacarídeo da raiz de Lótus (Nelumbo nucifera). Rev. Bras. Cien. Farm., São Paulo, v.40, n.3, p.425-431, 2004.

SEMDE, R.; AMIGHI, K.; PIERRE, D.; DEVLEESCHOUWER, M. J.; MOES, A. J. Leaching of pectin from mixed pectin: insoluble polymer films intended for colonic drug delivery. Int. J. Pharm., Amsterdam, v.174, n.1-2, p.233-241, 1998.

SINHA, V.R.; KUMRIA, R. Microbially triggered drug delivery to the colon. Eur. J. Pharm. Sci., Amsterdam, v.18, n.1 p.3-18, 2003.

SINHA, V.R.; KUMRIA, R. Polysaccharides in colonspecific drug delivery. Int. J. Pharm., Amsterdam, v.224, n.1-2, p.19-38, 2001.

Recebido para publicação em 13 de junho de 2007 Aceito para publicação em 11 de dezembro de 2007 